Для цитирования: Овчинников А.А., Султанова А.Н., Станкевич А.С., Наров М.Ю., Чут У.Ю., Иоаниди Д.К., Луговенко В.А. Особенности аддиктивных расстройств и аффективной симптоматики при шизофрении. Cuбирский вестник психиатрии и наркологии. 2021; 2 (111): 23-31. https://doi.org/10.26617/1810-3111-20212(111)-23-31

\title{
Особенности аддиктивных расстройств и аффективной симптоматики при шизофрении
}

\section{Овчинников А.А. ${ }^{1}$, Султанова А.Н. ${ }^{1,2}$, Станкевич А.C. ${ }^{3}$, Наров М.Ю.', Чут У.Ю.', Иоаниди Д.К. ${ }^{1}$, Луговенко В.А. ${ }^{4}$}

\author{
${ }^{1}$ ФГБОУ ВО «Новосибирский государственный медицинский университет» Минздрава России \\ Россия, 630091, Новосибирск, Красный проспект, 52 \\ 2 ФГБОУ ВО «Новосибирский государственный университет экономики и управления» \\ Россия, 630099, Новосибирск, ул. Каменская, 56 \\ ${ }^{3}$ ГАУССО НСО «Успенский психоневрологический интернат» \\ Россия, 633146, Новосибирская область, Мошковский район, с. Успенка, ул. Поросская, 1 \\ ${ }^{4}$ Медииинский иентр «Синеглазка» \\ Россия, 630112, Новосибирск, ул. Фрунзе, 61
}

\section{PEЗЮME}

Актуальность. Аффективная и аддиктивная патология широко распространена среди пациентов с расстройствами шизофренического спектра; коморбидность расстройств ограничивает социальную и профессиональную активность пациентов, неблагоприятно сказывается на семейном и социальном статусе, усугубляет тяжелые явления госпитализма и инвалидизации. Цель: выявление особенностей аддиктивных расстройств и аффективной симптоматики при шизофрении. Материалы и методы. Исследование проводилось в течение двух лет (2019-2020 гг.). Основную выборку составили пациенты (n=34) с диагностированной по МКБ-10 шизофренией (F20), с различными клиническими формами и длительностью заболевания, с преобладанием мужчин (n=32) по сравнению с женщинами $(\mathrm{n}=2)$, средний возраст обследованных составил $28,5 \pm 9,8$ года. Для диагностики аффективной симптоматики использованы четыре стандартизованных психометрических метода: Госпитальная шкала тревоги и депрессии (Zigmond A.S., Snaith R.P., 1983 - HADS), Опросник для определения уровня и типа одиночества (Корчагина C.Г., 2008), Шкала диагностики агрессивности и враждебности Кука-Медли (Cook W.W., Medley D.M., 1954), Шкала безнадежности А. Бека (Beck A.T. et al., 1974 - BНI). Изучение склонности к зависимостям проводилась по Методике диагностики склонности к различным зависимостям (Лозовая Г.В., 2007). Результаты. Выявлены высокая степень склонности к трем видам зависимости (любовная, лекарственная, здорового образа жизни), низкий и субклинический уровни выраженности тревоги и депрессии, легкая и средняя выраженность безнадежности, глубокое переживание одиночества, средняя степень наклонности к агрессия с тенденцией к низким показателям. Также была получена достоверная связь между одиночеством, религиозной зависимостью и сексуальной аддикцией.

Ключевые слова: шизофрения, психиатрическая коморбидность, аффективные и аддиктивные (зависимости) расстройства, тревога, депрессия, безнадежность, одиночество, агрессия и враждебность.

\section{ВВЕДЕНИЕ}

Шизофрения - это серьезное психическое расстройство, которое может иметь тяжелые социально-психологические последствия для пациентов и их семей [1]. Пациенты с различными симптомами, клиническими формами и типами течения шизофрении зачастую характеризуются повышенным риском бездомности [2], высоким уровнем употребления психоактивных веществ (ПАВ) [3] и резко сниженной ожидаемой продолжительностью жизни по сравнению с общей популяцией населения [4]. Люди с шизофренией также испытывают низкое качество жизни, трудности в социальном принятии, в реализации значимых жизненных целей из-за коморбидных симптомов тревоги и депрессии, вызывающих низкую активность и мотивацию $[5,6]$, а также подвержены влиянию дискриминации и социальной стигматизации [7]. Важно отметить, что пациенты с шизофренией имеют повышенный риск развития депрессивных симптомов по сравнению с уже высокой пожизненной распространенностью депрессии в общей популяции [8]. Многие исследователи отмечают, что депрессивная симптоматика диагностируется на всех стадиях течения шизофрении $[9,10]$. Наиболее актуальными и дискуссионными проблемами в обсуждении проявлений коморбидности является то, что депрессивные симптомы высоко коррелируют с риском суицида $[11,12]$. 
В то же время депрессивные пациенты предрасположены к более высокому риску развития психоза, причем депрессия часто наблюдается у людей с высоким риском развития шизофрении ещё до появления психотических симптомов [13, $14,15]$. Кроме того, формирование психотических симптомов при депрессии, рассматриваемой как отдельный клинический подтип депрессии, включенный в МКБ-10 как депрессивный эпизод тяжелой степени с психотипическими симптомами, связано с наличием галлюцинаций и бреда [16, 17]. Эта взаимная коморбидность шизофрении и депрессии предполагает потенциальное совпадение как этиологических, так и патофизиологических механизмов развития клинических проявлений заболевания. В некоторых работах прослеживается идея о том, что депрессивные симптомы являются синдромальным конструктом при шизофрении $[18,19]$. Однако следует учитывать, что депрессивные симптомы при шизофрении могут являться побочным эффектом нейролептической терапии, или вторичным осложнением по отношению к другим сопутствующим заболеваниям, таким как злоупотребление ПАВ, или психологической реакцией на последствия расстройства $[20$, 21, 22].

Злоупотребление психоактивными веществами при шизофрении - одна из стержневых проблем социальной дезадаптации, снижения комплаентности и эффективности терапии [23]. В ряде исследований приводятся данные, что пациенты с шизофренией в 4,6 раза чаще имеют расстройства, связанные с употреблением ПАВ, чем лица без психических заболеваний (в 3 раза выше при приеме алкоголя, в 6 раз выше при употреблении наркотиков) [24, 25]. Пациенты с шизофренией обычно используют одно или несколько веществ, включая никотин, алкоголь, каннабис, кокаин и амфетамины $[26,27]$. Никотин наиболее часто используется в популяции пациентов с шизофренией, причем уровень его употребления колеблется от $70 \%$ до $90 \%$ по сравнению с $26 \%$ в общей популяции [28]. Алкоголь является следующим частым веществом злоупотребления среди коморбидных пациентов, за которым следуют каннабис или кокаин, хотя употребление амфетаминов, опиатов, галлюциногенов, седативных средств, кофеина встречается не менее часто. Не следует оставлять без внимания и тот факт, что определяющим стимульным мотивом для употребления является доступность ПАВ [29, 30]. Анализ и оценка статистических показателей распространенности злоупотребления ПАВ выявил, что пациенты с шизофренией злоупотребляют кокаином от $15 \%$ до $50 \%$ случаев, амфетамином - от $2 \%$ до $25 \%$, алкоголем - от $20 \%$ до $60 \%$ и каннабисом - от $12 \%$ до $42 \%[31,32]$.
Пациенты с шизофренией и сопутствующими расстройствами употребления ПАВ имеют худший прогноз течения с прогрессированием болезни, низкую приверженность к лечению, юридические и социально-правовые проблемы в реализации жизнедеятельности и широкий круг соматических заболеваний $[33,34]$. Кроме того, больные шизофренией и коморбидным расстройством потребления ПАВ имеют больше причин и показаний для частых и длительных стационарных госпитализаций [35] и высокий суицидальный риск $[36,37]$. Коморбидность между двумя заболеваниями объясняется рядом процессов. Во-первых, эти два расстройства имеют общие этиологические факторы развития, в том числе риск генетической предрасположенности; во-вторых, одно нарушение является пусковым механизмом, провоцирующим развитие другого [38, 39]. Теоретические и практические аспекты изучения аддиктивных и аффективных расстройств у больных шизофренией актуализируют специфику восприятия болезни и недостатки разработанности данного вопроса.

\section{ЦЕЛЬ ИССЛЕДОВАНИЯ}

Выявить особенности аддиктивных расстройств и аффективной симптоматики при шизофрении.

\section{МАТЕРИАЛЫ И МЕТОДЫ}

Исследование проводилось в течение двух лет (2019-2020 гг.). Основную выборку составили пациенты $(\mathrm{n}=34)$ с диагностированной по МКБ-10 шизофренией (F20), с различными клиническими формами и длительностью заболевания, с преобладанием мужчин (n=32) по сравнению с женщинами $(\mathrm{n}=2)$, средний возраст обследованных составил $28,5 \pm 9,8$ года. Для диагностики аффективной симптоматики использованы четыре стандартизованных психометрических метода: Госпитальная шкала тревоги и депрессии (Zigmond A.S., Snaith R.P., 1983 - HADS), Опросник для определения уровня и типа одиночества (Корчагина С.Г., 2008), Шкала диагностики агрессивности и враждебности Кука-Медли (Cook W.W., Medley D.M., 1954), Шкала безнадежности А. Бека (Beck A.T. et al., 1974 - BНI). Изучение склонности к зависимостям проводилась по Методике диагностики склонности к различным зависимостям (Лозовая Г.В., 2007).

Статистическая обработка полученных результатов осуществлялась на персональном компьютере с использованием стандартных методов подсчета средних величин и стандартного отклонения. Все вычисления проводились с помощью пакета статистического программного обеспечения StatSoft «Statistica 10» с определением описательной статистики (средние величины, стандартное отклонение). 
Для выявления различий между группами по каждому показателю определялись с помощью Uкритерия Манна-Уитни, для определения связи между факторами был задействован коэффициент ранговой корреляции Спирмена (r).

\section{РЕЗУЛЬТАТЫ И ОБСУЖДЕНИЕ}

На начальном этапе участникам исследования была предложена для заполнения анкета, использованная как средство нивелирования беспокойства, внутреннего напряжения и страха, а также создания благоприятного коммуникативного пространства для смягчения тревожного ожидания перед диагностическим экспериментальнопсихологическим исследованием. В выборке пациентов с диагнозом шизофрении уточнена длительность течения заболевания (рис. 1).

Ведущие жалобы, предъявляемые пациентами, включают навязчивый и устойчивый страх за будущее и тревожно-соматический компонент в виде ипохондрической фобии.
Феномен психической дезадаптации в рамках течения шизофрении подробно описал З.И. Кекелидзе [40]. Такие пациенты боятся осуждения со стороны близких и знакомых людей, полностью охвачены чувством непонимания и одиночества от неприятия обществом. Также пациенты со сложностями в общении и профессиональном самоопределении испытывают страх, что не смогут устроиться на работу, а мужчины переживают, что не смогут обеспечить семью по причине потери постоянного источника дохода в конечном итоге. Отсюда вытекает необходимость регулярной поддержки со стороны социальных служб на амбулаторном этапе, что позволит минимизировать страх после выписки больных шизофренией с длительными сроками госпитализации в психиатрических стационарах для лучшей адаптации к внебольничной среде и достижения наиболее глубоких ремиссий в последующем.

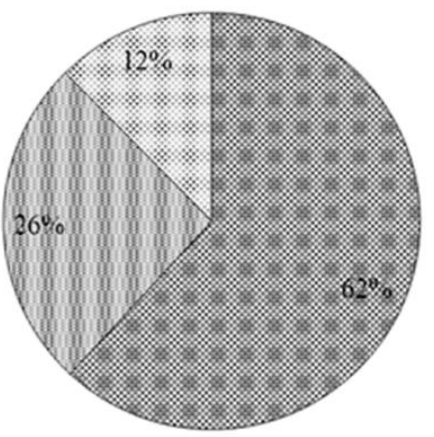

Менее 10 лет

๗ Более 10 лет

응 20 лет

\section{Р и с у н о к 1. Сравнительный анализ длительности болезни у обследованных больных шизофренией (\%)}

Как видно из рисунка 1 , большинство пациентов в нашей выборке составили те, у кого длительность болезни превышала 10 лет $(62 \%, \mathrm{n}=21)$. В то время как длительность шизофренического процесса от 20 лет и более показала самую низкую частоту встречаемости $(12 \%, \mathrm{n}=4)$.

Следующим этапом стало психодиагностическое исследование эмоционального состояния больных шизофренией для изучения эмоционально-аффективных нарушений. Ниже приведены результаты средних значений и стандартных отклонений, однако достоверных различий между группами по данным показателям не обнаружено.
Согласно приведенным в таблице 1 данным, у пациентов с шизофренией выявлены низкий $(2,7 \pm 3,2 ; 5,3 \pm 3,1)$ и субклинический $(9,8 \pm 4,2$; $9,3 \pm 4,3)$ уровни выраженности тревоги и депрессии, а также легкая $(5,3 \pm 1,5)$ и средняя $(9,0 \pm 4,2)$ выраженность безнадежности. Полученные результаты являются вариантом нормы, однако стоит обратить внимание на эти показатели, так как данные состояния могут перейти по степени тяжести в выраженные психотические проявления.

Зарегистрирована средняя $(28 \pm 5,7)$ степень наклонности к агрессии с тенденцией к низким показателям (рис. 2).

Т а б л и ц а $\begin{gathered}\text { 1. Средние значения и стандартные отклонения уровней тревоги, депрессии } \\
\text { и безнадежности у обследованных больных шизофренией }\end{gathered}$
\begin{tabular}{|l|c|c|c|c|}
\hline \multirow{3}{*}{ Шкала } & \multicolumn{3}{|c|}{ Общая группа обследованных пациентов с шизофренией } \\
\cline { 2 - 5 } & \multicolumn{3}{|c|}{$\begin{array}{c}\text { показатели субклинически выраженной } \\
\text { депрессии, тревоги и безнадежности }\end{array}$} & $\begin{array}{c}\text { нормативные показатели депрессии, } \\
\text { тревоги и безнадежности }\end{array}$ \\
\cline { 2 - 5 } & Среднее значение & Стандартное отклонение & Среднее значение \\
(M) & (SD) & 2,7 & $\begin{array}{c}\text { Стандартное отклонение } \\
\text { (SD) }\end{array}$ \\
\hline Тревога & 9,8 & 4,2 & 5,3 & 2,3 \\
\hline Безнадежность & 9 & 4,2 & 5,3 & 1,5 \\
\hline Депрессия & 9,3 & 4,3 & & 3,1 \\
\hline
\end{tabular}



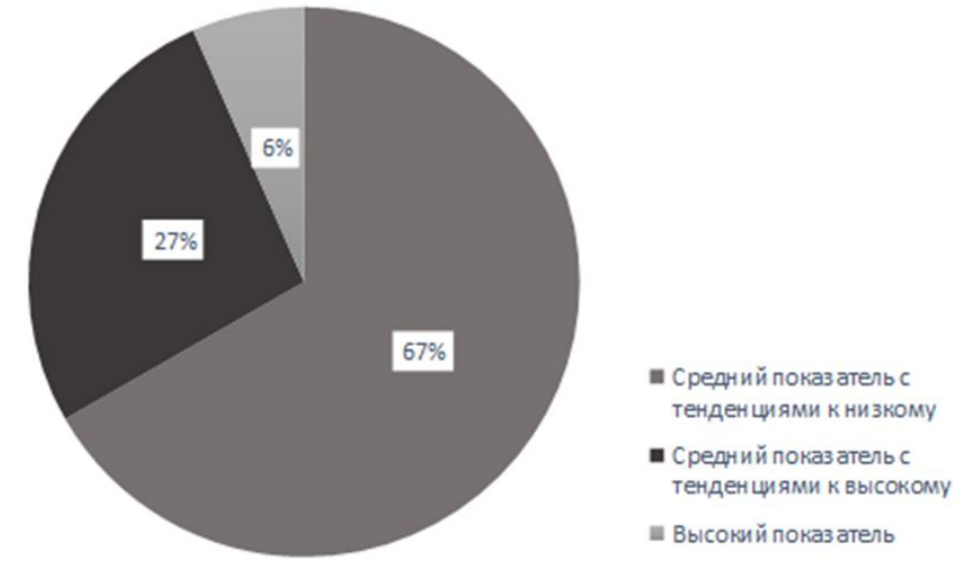

Р и с у н о к 2. Сравнительный анализ выраженности агрессии у обследованных больных шизофренией (\%)

Действительно, имеются достаточные основания говорить о высокой распространенности среди пациентов с шизофренией агрессии, мотивами которой могут служить бредовые механизмы, бесконтрольность эмоционального реагирования и расторможенность влечений в структуре негативно-личностных образований. В острых стадиях болезни они могут быть озлобленными и агрессивными в связи с неверной интерпретацией действительной реальности и поведения окружающих, поэтому данный вопрос также требует тщательного изучения.

Как показано на рисунке 3 , все пациенты чувствуют себя одинокими (100\%), разница заключа- ется только в степени выраженности этого чувства: 73,5\% (n=25) испытывают глубокие переживания одиночества, 20,6\% (n=7) подвержены не очень глубоким переживаниям, связанным с одиночеством. Лишь у незначительной части $(5,9 \%$, $\mathrm{n}=2$ ) больных наблюдаются очень глубокие переживания отрыва от внешнего мира, брошенности и одиночества. В ходе исследования с помощью коэффициента ранговой корреляции Спирмена (r) обнаружена статистически достоверная связь между переживанием, связанным с одиночеством ввиду ограничения/отсутствия социальных контактов, и длительностью течения шизофрении $(\mathrm{r}=0,4)$.

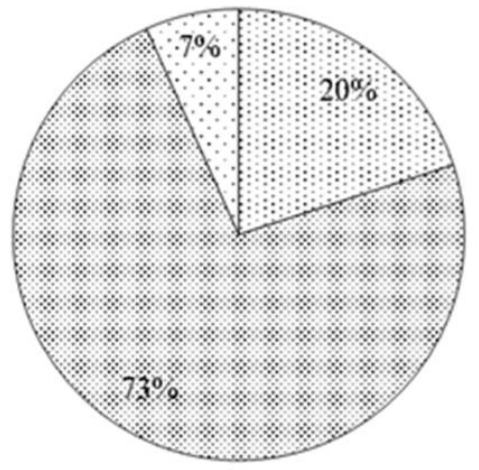

曰Неглобокие переживания
одиночества
凤 Глубокие переживания
одиночества
Фочень глубокие
переживания
одиночеста

Р и с у н о к 3. Сравнительный анализ выраженности одиночества у обследованных больных шизофренией (\%)

Далее было проведено тестирование пациентов по Методике диагностики склонности к различным зависимостям (Лозовая Г.В., 2007), выявляющей 13 видов зависимостей. Аддиктивное (зависимое) поведение представляет собой попытку бегства от реальной жизни при помощи изменения своего психического состояния, обеспечивающего мнимую безопасность и эмоциональный комфорт. Несмотря на кажущиеся внешние различия, зависимые формы поведения име- ют принципиально схожие психологические глубинные проблемы. По результатам тестирования выявлена высокая склонность к некоторым зависимостям, в частности к любовной $(88,2 \%)$ и лекарственной $(88,2 \%)$ зависимостям и чрезмерному вниманию к поддержанию здорового образа жизни (73,5\%). С помощью коэффициента Спирмена установлено, что игровая зависимость нарастает с увеличением длительности шизофрении $(\mathrm{r}=0,3)$. 
Завершающим этапом исследования явилось выявление статистически достоверной связи между аддикциями и одиночеством, депрессией и агрессией. На рисунке 4 представлена корреляционная плеяда значимых взаимосвязей основной выборки, учитывались результаты при р<0,05.

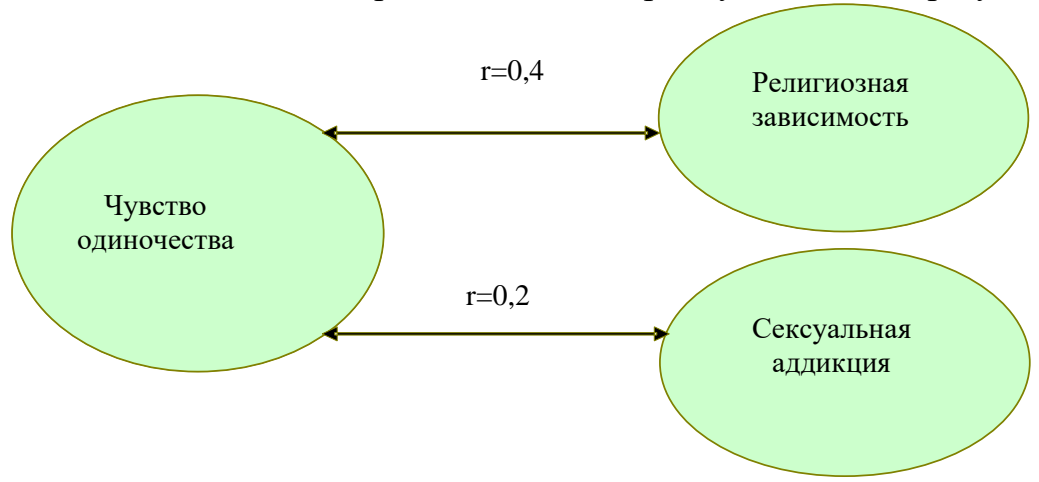

Р и с у н о к 4. Корреляционная плеяда значимых взаимосвязей, выявленных по результатам исследования больных шизофренией с аддиктивным поведением и чувством одиночества

Как продемонстрировано на рисунке 4, в соответствии со значениями коэффициента корреляционной связи, несомненно, имеют место умеренно выраженная положительная связь между одиночеством и религиозной зависимостью $(\mathrm{r}=0,4)$ и слабая положительная связь между одиночеством и сексуальной аддикцией $(\mathrm{r}=0,2)$.

Мы предполагаем, что восприятие действительности с позиций религиозного мировоззрения помогает пациентам с шизофренией чувствовать себя менее одинокими и брошенными, веря в достижение своих целей. Знание о том, что есть Бог, который всегда рядом, облегчает эмоциональное переживание одиночества, дает опору и стабильность в жизни, пусть даже иллюзорную, привносит ритуалы и стереотипы в повседневные дела, что создает искаженное представление о контроле за собственной жизнью. Вовлечение в компенсирующую сферу религиозного сообщества и участие в религиозных церемониях и службах также способно снизить чувство одиночества, так как при богослужении каждый верующий испытывает сопричастность с присутствующими, ощущение целостности с другими людьми. Активные и адаптивные поведенческие стратегии преодоления болезни у пациентов с остаточной шизофренией связаны со здоровой духовной, религиозной или личной системой убеждений. Среди психотической симптоматики у больных шизофренией описаны симптомы, квалифицируемые в психиатрической литературе как «бред религиозного содержания» [41].

Сексуальную жизнь больных шизофренией можно считать осложненной в связи с нарушением эмоционально-волевой сферы и интерперсональных коммуникаций, что может повлечь за собой особенно глубокие переживания нарастающего чувства одиночества. Среди клинических проявлений шизофрении важную роль играет нарастание аутизма, отчужденности. Это изменяет отношение к окружающему, сужает личностные связи, затрудняет установление и поддержание половых контактов. При лечении данного контингента больных требуется учитывать основные негативные проявления болезни, которые препятствуют активной социальной и личной жизни. В первую очередь решается задача смягчения психопатологической симптоматики с применением нейролептических препаратов мягкого действия, антидепрессантов в сочетании с ноотропными средствами. Заметное послабление аффективных, сенестопатически-ипохондрических и обсессивных расстройств позволяет начать психотерапию, направленную на разубеждение больных в тяжести их состояния, создание лечебной перспективы, активацию личностных ресурсов в сфере социальных и сексуальных контактов.

\section{ВЫВОДЫ И ЗАКЛЮЧЕНИЕ}

В выборке пациентов с различными клиническими формами и длительностью шизофрении выявлена высокая степень склонности к зависимостям, таким как любовная $(88,2 \%)$, лекарственная $(88,2 \%)$, здорового образа жизни $(73,5 \%)$.

Обнаружены низкий $(2,7 \pm 3,2 ; 5,3 \pm 3,1)$ и субклинический $(9,8 \pm 4,2 ; 9,3 \pm 4,3)$ уровни выраженности тревоги и депрессии соответственно, а также легкая $(5,3 \pm 1,5)$ и средняя $(9,0 \pm 4,2)$ выраженность безнадежности.

Установлены структура и степень выраженности эмоционально-аффективных нарушений, что позволяет констатировать глубокое переживание одиночества, в то время как агрессия имеет среднюю $(28 \pm 5,7)$ степень наклонности с тенденцией к низким показателям.

Зафиксированы умеренно выраженная положительная связь между одиночеством и религиозной зависимостью $(\mathrm{r}=0,4)$ и слабая положительная связь между одиночеством и сексуальной аддикцией $(\mathrm{r}=0,2)$. Обращение к религии помогает менее пессимистично ощущать себя одинокими, брошенными и ненужными. Сексуальная жизнь осложнена нарушениями в эмоционально-волевой и психосексуальной сферах и интерперсональнокоммуникативной деятельности. 
Получена статистически достоверная связь между одиночеством $(\mathrm{r}=0,4)$, игровой зависимостью $(\mathrm{r}=0,3)$ и длительностью шизофренического процесса с помощью коэффициента Спирмена.

Таким образом, в ходе исследования выявлено, что пациенты с различными клиническими формами и длительностью шизофрении склонны к высокой степени зависимости - любовной, лекарственной и чрезмерному вниманию к здоровому образу жизни. Кроме того, для них характерны: 1) низкий и субклинический уровни выраженности тревоги и депрессии, 2) легкая и средняя выраженность безнадежности, глубокое переживание одиночества, 3) отсутствие депрессивных состояний/наклонности, 4) средний показатель степени выраженности агрессии, 5) статистически достоверные связи между религиозной и сексуальной аддикцией и одиночеством. Полученные результаты свидетельствуют, что существуют особенности как аффективной симптоматики (в виде проявлений тревоги и депрессии), так и аддиктивных расстройств (в виде склонности к определенным видам зависимости), а также степени выраженности одиночества, депрессии, агрессии. Как и следовало ожидать, результаты исследования нуждаются в доработке, увеличении количества выборок, расширении базы данных для уточнения и конкретизации объекта исследования, тем не менее уже на настоящий момент являются достаточно подкрепленными интерпретацией полученных данных.

\section{КОНФЛИКТ ИНТЕРЕСОВ}

Авторы заявляют об отсутствии явных и потенциальных конфликтов интересов в связи с публикацией данной статьи.

\section{ИСТОЧНИК ФИНАНСИРОВАНИЯ}

Авторы заявляют об отсутствии спонсорских или иных финансовых источников при проведении исследования.

\section{СООТВЕТСТВИЕ ПРИНЦИПАМ ЭТИКИ}

Исследование одобрено ЛЭК при ФГБОУ ВО «Новосибирский государственный медицинский университет» Минздрава России (протокол № 136 от 17 июня 2021 г.).

\section{ЛИТЕРАТУРА/REFERENCES}

1. Knapp M, Mangalore R, Simon J. The global costs of schizophrenia. Schizophr Bull. 2004;30(2):27993. doi: 10.1093/oxfordjournals.schbul.a007078. PMID: 15279046.

2. Folsom D, Jeste DV. Schizophrenia in homeless persons: a systematic review of the literature. Acta Psychiatr Scand. 2002 Jun;105(6):404-13. doi: 10.1034/j.1600-0447.2002.02209.x. PMID: 12059843.

3. Fowler IL, Carr VJ, Carter NT, Lewin TJ. Patterns of current and lifetime substance use in schizophrenia. Schizophr Bull. 1998;24(3):443-55. doi: 10.1093/oxfordjournals.schbul.a033339. PMID: 9718636.
4. Brown S, Inskip H, Barraclough B. Causes of the excess mortality of schizophrenia. Br J Psychiatry. 2000 Sep;177:212-7. doi: 10.1192/bjp.177.3.212. PMID: 11040880.

5. Гуткевич Е.В., Семке А.В. Семейные механизмы адаптации при шизофренических расстройствах(на примере популяции Сибирского региона). Сибирский вестник психиатрии и наркологии. 2012. № 3 (72). С. 12-17. Gutkevich EV, Semke AV. Family mechanisms of adaptation in schizophrenic disorders (on example of population of the Siberian region). Siberian Herald of Psychiatry and Addiction Psychiatry. 2012;3(72):12-17 (in Russian).

6. Rosenheck R, Leslie D, Keefe R, McEvoy J, Swartz M, Perkins D, Stroup S, Hsiao JK, Lieberman J; CATIE Study Investigators Group. Barriers to employment for people with schizophrenia. Am J Psychiatry. 2006 Mar;163(3):411-7. doi: 10.1176/appi.ajp.163.3.411. PMID: 16513861.

7. Dickerson FB, Sommerville J, Origoni AE, Ringel NB, Parente F. Experiences of stigma among outpatients with schizophrenia. Schizophr Bull. 2002;28(1):143-55.

doi: 10.1093/oxfordjournals.schbul.a006917. PMID: 12047014.

8. Buckley PF, Miller BJ, Lehrer DS, Castle DJ. Psychiatric comorbidities and schizophrenia. Schizophr Bull. 2009;35(2):383-402. doi: $10.1093 / \mathrm{schbul} / \mathrm{sbn} 135$

9. Häfner H, Maurer K, An der Heiden W. Schizophrenie - eine einheitliche Krankheit?: Ergebnisse aus 25 Jahren ABC-Studie [Schizophrenia - a disorder in its own right?: results from 25 years of the ABC study]. Nervenarzt. 2013 Sep;84(9):1093-4, 1096-103. German. doi: 10.1007/s00115-013-37886. PMID: 23695002.

10. Sönmez N, Røssberg JI, Evensen J, Barder HE, Haahr U, Ten Velden Hegelstad W, Joa I, Johannessen JO, Langeveld H, Larsen TK, Melle I, Opjordsmoen S, Rund BR, Simonsen E, Vaglum P, McGlashan T, Friis S. Depressive symptoms in first-episode psychosis: a 10-year follow-up study. Early Interv Psychiatry. 2016 Jun;10(3):227-33. doi: 10.1111/eip.12163. Epub 2014 Jun 23. PMID: 24956976.

11. Upthegrove R, Birchwood M, Ross K, Brunett K, McCollum R, Jones L. The evolution of depression and suicidality in first episode psychosis. Acta Psychiatr Scand. 2010 Sep;122(3):211-8. doi: 10.1111/j.1600-0447.2009.01506.x. Epub 2009 Nov 18. PMID: 19922525.

12. Andriopoulos I, Ellul J, Skokou M, Beratis S. Suicidality in the "prodromal" phase of schizophrenia. Compr Psychiatry. 2011 Sep-Oct;52(5):479-85. doi: 10.1016/j.comppsych.2010.10.011. Epub 2010 Dec 23. PMID: 21185016.

13. Ohayon MM, Schatzberg AF. Prevalence of depressive episodes with psychotic features in the general population. Am J Psychiatry. 2002 
Nov;159(11):1855-61.

doi:

10.1176/appi.ajp.159.11.1855. PMID: 12411219.

14. Häfner $\mathrm{H}$, Maurer $\mathrm{K}$, Trendler $\mathrm{G}$, an der Heiden $\mathrm{W}$, Schmidt M, Könnecke R. Schizophrenia and depression: challenging the paradigm of two separate diseases - a controlled study of schizophrenia, depression and healthy controls. Schizophr Res. 2005 Sep 1;77(1):11-24. doi: 10.1016/j.schres.2005.01.004 PMID: 16005381.

15. Schothorst PF, Emck C, van Engeland H. Characteristics of early psychosis. Compr Psychiatry. 2006 NovDec;47(6):438-42. 10.1016/j.comppsych.2006.03.003. Epub 2006 May 3 PMID: 17067866.

16. Park SC, Lee HY, Sakong JK, Jun TY, Lee MS, Kim JM, Kim JB, Yim HW, Park YC. Distinctive Clinical Correlates of Psychotic Major Depression: The CRESCEND Study. Psychiatry Investig. 2014 Jul;11(3):281-9. doi: 10.4306/pi.2014.11.3.281. Epub 2014 Jul 21. PMID: 25110501; PMCID: PMC4124187.

17. Gournellis R, Oulis P, Howard R. Psychotic major depression in older people: a systematic review. Int J Geriatr Psychiatry. 2014 Aug;29(8):789-96. doi: 10.1002/gps.4065. PMID: 25191689.

18. an der Heiden W, Könnecke R, Maurer K, Ropeter D, Häfner H. Depression in the long-term course of schizophrenia. Eur Arch Psychiatry Clin Neurosci. 2005 Jun;255(3):174-84. doi: 10.1007/s00406-0050585-7. PMID: 15995901.

19. Chiappelli J, Kochunov P, DeRiso K, Thangavelu K, Sampath H, Muellerklein F, Nugent KL, Postolache TT, Carpenter WT Jr, Hong LE. Testing trait depression as a potential clinical domain in schizophrenia. Schizophr Res. 2014 Oct;159(1):243-8. doi: 10.1016/j.schres.2014.08.003. Epub 2014 Aug 27. PMID: 25171855; PMCID: PMC4177287.

20. Turkington A, Mulholland CC, Rushe TM, Anderson R, McCaul R, Barrett SL, Barr RS, Cooper SJ. Impact of persistent substance misuse on 1-year outcome in first-episode psychosis. Br J Psychiatry. 2009 Sep;195(3):242-8. doi: 10.1192/bjp.bp.108.057471. PMID: 19721115.

21. Clarke DM, Kissane DW. Demoralization: its phenomenology and importance. Aust N Z J Psychiatry. 2002 Dec;36(6):733-42. doi: 10.1046/j.14401614.2002.01086.x. PMID: 12406115.

22. Ветлугина Т.П., Лобачева О.А., Семке А.В., Сергеева С.А., Эпштейн О.И., Мальцева Ю.Л. Способ лечения больных шизофренией. Патент на изобретение RU $2415666 \mathrm{C} 1,10.04 .2011$. Заявка № 2009143445/15 от 24.11.2009. Vetlugina TP, Lobacheva OA, Semke AV, Sergeeva SA, Epstein OI, Maltseva YuL. A method of treating patients with schizophrenia. Patent for invention RU $2415666 \mathrm{C} 1$, 10.04.2011. Application no. 2009143445/15 dated 24.11.2009 (in Russian).

23. Дмитриева Е.Г., Даниленко О.А., Корнетова Е.Г., Семке А.В., Лобачева О.А., Гуткевич Е.В., Каткова М.Н. Комплаенс и его влияние на адап- тацию пациентов с шизофренией. Сибирский вестник психиатрии и наркологии. 2014. № 3 (84). C. 18-23. Dmitrieva EG, Danilenko OA, Kornetova EG, Semke AV, Lobacheva OA, Gutkevich EV, Katkova MA. Influence of compliance on adaptation of schizophrenic patients. Siberian Herald of Psychiatry and Addiction Psychiatry. 2014;3(84):18-23 (in Russian).

24. Oranje B, Geyer MA, Bocker KB, Leon Kenemans J, Verbaten MN. Prepulse inhibition and P50 suppression: commonalities and dissociations. Psychiatry Res. 2006 Aug 30;143(2-3):147-58. doi: 10.1016/j.psychres.2005.11.002. Epub 2006 Jul 31. PMID: 16879870.

25. Düring S, Glenthøj BY, Andersen GS, Oranje B. Effects of dopamine D2/D3 blockade on human sensory and sensorimotor gating in initially antipsychotic-naive, first-episode schizophrenia patients. Neuropsychopharmacology. 2014 Dec;39(13):3000-8. doi: 10.1038/npp.2014.152. Epub 2014 Jul 23. PMID: 24954063; PMCID: PMC4229570.

26. Ross CA, Margolis RL, Reading SA, Pletnikov M, Coyle JT. Neurobiology of schizophrenia. Neuron. 2006 Oct 5;52(1):139-53. doi: 10.1016/j.neuron.2006.09.015. PMID: 17015232.

27. Pješčić KD, Nenadović MM, Jašović-Gašić $M$, Trajković G, Kostić M, Ristić-Dimitrijević R. Influence of psycho-social factors on the emergence of depression and suicidal risk in patients with schizophrenia. Psychiatr Danub. 2014 Sep;26(3):226-30. PMID: 25191769.

28. Fatemi SH, Stary JM, Hatsukami DK, Murphy SE. A double-blind placebo-controlled cross over trial of bupropion in smoking reduction in schizophrenia. Schizophr Res. 2005 Jul 15;76(2-3):353-6. doi: 10.1016/j.schres.2005.02.021. Epub 2005 Apr 19. PMID: 15949668.

29. Rush B, Koegl CJ. Prevalence and profile of people with co-occurring mental and substance use disorders within a comprehensive mental health system. Can J Psychiatry. 2008 Dec;53(12):810-21. doi: 10.1177/070674370805301207. PMID: 19087479.

30. Mueser KT, Essock SM, Drake RE, Wolfe RS, Frisman L. Rural and urban differences in patients with a dual diagnosis. Schizophr Res. 2001 Mar 1;48(1):93-107. doi: 10.1016/s09209964(00)00065-7. PMID: 11278157.

31. Tiet QQ, Mausbach B. Treatments for patients with dual diagnosis: a review. Alcohol Clin Exp Res. 2007 Apr;31(4):513-36. doi: 10.1111/j.15300277.2007.00336.x. PMID: 17374031.

32. Griffin ML, Kolodziej ME, Weiss RD. Measuring principal substance of abuse in comorbid patients for clinical research. Addict Behav. 2009 Oct;34(10):826-9.

doi: 10.1016/j.addbeh.2009.03.002. Epub 2009 Mar 6. PMID: 19285810; PMCID: PMC2720415.

33. Bennett ME, Gjonbalaj S. The problem of dual diagnosis. In: Adult Psychopathology and Diagnosis, 
5th ed. Edited by Hersen M, Beidel D. New York, Wiley, 2007.

34. Дубровская В.В., Корнетова Е.Г., Семке А.В., Иванова С.А., Аржаник М.Б., Головаха Н.Э. Клиническая и социальная адаптация у пациентов с шизофренией и сопутствующим метаболическим синдромом. Современная терапия психических расстройств. 2019. № 2. С. 17-21. Dubrovskaya VV, Kornetova EG, Semke AV, Ivanova SA, Arzhanik MB, Golovakha NE. Clinical and social adaptation in patients with schizophrenia and concomitant metabolic syndrome. Modern Therapy of Mental Disorders. 2019;2:1721 (in Russian).

35. Swofford CD, Scheller-Gilkey G, Miller AH, Woolwine B, Mance R. Double jeopardy: schizophrenia and substance use. Am J Drug Alcohol Abuse. 2000 Aug;26(3):343-53. doi: 10.1081/ada100100248. PMID: 10976661.

36. Potvin S, Stip E, Roy JY. Clozapine, quetiapine and olanzapine among addicted schizophrenic patients: towards testable hypotheses. Int Clin Psychopharmacol. 2003 May; 18(3):121-32. doi: 10.1097/01.yic.0000063501.97247.38. PMID: 12702890.

37. Моор Л.В., Рахмазова Л.Д. Клинико-динамическая характеристика и факторы риска панических расстройств. Сибирский вестник психиатрии и наркологии. 2019. № 3 (104). C. 14-20. Moor LV, Rakhmazova LD. Clinical-dynamic characteristics and risk factors for panic disorders. Siberian Herald of Psychiatry and Addiction Psychiatry. 2019;3(104):14-20. https://doi.org/10.26617/1810-3111-2019-3(104)-14-20

38. Корнетова Е.Г., Дмитриева Е.Г., Тигунцев В.В., Гончарова А.А., Полежаев П.К., Иванова С.А.,
Семке А.В. Гиперпролактинемия у больных шизофренией, получающих галоперидол и рисперидон: клинико-социальный аспект. Сибирский вестник психиатрии и наркологии. 2019. № 2 (103). C. 90-97. Kornetova EG, Dmitrieva EG, Tiguntsev VV, Goncharova AA, Polezhaev PK, Ivanova SA, Semke AV. Hyperprolactinemia in patients with schizophrenia receiving haloperidol and risperidone: clinical-social features. Siberian Herald of Psychiatry and Addiction Psychiatry. 2019;2(103):90-97 (in Russian). https://doi.org/10.26617/1810-3111-2019-2(103)-90-97

39. Manseau MW, Goff DC. Cannabinoids and Schizophrenia: Risks and Therapeutic Potential. Neurotherapeutics. 2015 Oct;12(4):816-24. doi: 10.1007/s13311-015-0382-6. PMID: 26311150; PMCID: PMC4604190.

40. Кекелидзе 3.И., Пасынкова Ю.Г., Бедина И.А. Стигматизация больных шизофренией: методические рекомендации. М. : Изд-во ЗАО Юстицинформ, 2009. 28 с. Kekelidze ZI, Pasynkova YuG, Bedina IA. Stigmatization of patients with schizophrenia: guidelines. Moscow: Yustitsinform Publishing House, 2009:28.

41. Mohr S, Brandt PY, Borras L, Gilliéron C, Huguelet P. Toward an integration of spirituality and religiousness into the psychosocial dimension of schizophrenia. Am J Psychiatry. 2006 Nov;163(11):1952-9. doi: 10.1176/ajp.2006.163.11.1952. PMID: 17074947.

Поступила в редакцию 11.02.2021 Утверждена к печати 28.05.2021

Овчинников Анатолий Александрович - д.м.н., профессор, заведующий кафедрой психиатрии, наркологии, психотерапии и клинической психологии. SPIN-код 2228-5923. Author ID 705354. ORCID iD 0000-0003-1468-1620.

Султанова Аклима Накиповна - д.м.н., доцент кафедры психиатрии, наркологии, психотерапии и клинической психологии. ORCID iD 0000-0001-6420-6591.

Станкевич Анна Сергеевна - аспирант кафедры психиатрии, наркологии, психотерапии и клинической психологии. ORCID iD 0000-0001-5627-8245. stankevichann97@ mail.ru

Наров Михаил Юрьевич - д.м.н., профессор кафедры психиатрии, наркологии, психотерапии и клинической психологии. ORCID iD 0000-0001-7551-6333.2996458@mail.ru

Чут Ульяна Юрьевна - аспирант кафедры психиатрии, наркологии, психотерапии и клинической психологии. ORCID iD 0000-0001-7991-374X.uu_chut@mail.ru

Иоаниди Диана Константиновна - преподаватель кафедры психиатрии, наркологии, психотерапии и клинической психологии. ORCID iD 0000-0003-3103-2585. dion48@mail.ru

Луговенко Вероника Алексеевна - клинический психолог. ORCID iD 0000-0003-3894-5029. veronika060596@mail.ru

Султанова Аклима Накиповна, sultanova.aklima@yandex.ru

UDC 616.895.8:616.8-008.64:612.393.1:613.83

For citation: Ovchinnikov A.A., Sultanova A.N., Stankevich A.S., Narov M.Yu., Chut U.Yu., Ioanidi D.K., Lugovenko V.A. Features of addictive disorders and affective symptoms in schizophrenia. Siberian Herald of Psychiatry and Addiction Psychiatry. 2021; 2 (111): 23-31. https://doi.org/10.26617/1810-3111-2021-2(111)-23-31 


\title{
Features of addictive disorders and affective symptoms in schizophrenia
}

\section{Ovchinnikov A.A. ${ }^{1}$, Sultanova A.N. ${ }^{1,2}$, Stankevich A.S. ${ }^{3}$, Narov M.Yu. ${ }^{1}$, Chut U.Yu. ${ }^{1}$, loanidi D.K. ${ }^{1}$, Lugovenko V.A. ${ }^{4}$}

\author{
${ }^{I}$ Novosibirsk State Medical University of Ministry of Health of Russia \\ Krasnyy Avenue 52, 630091, Novosibirsk, Russian Federation \\ ${ }^{2}$ Novosibirsk State University of Economics and Management \\ Kamenskaya Street 56, 630099, Novosibirsk, Russian Federation \\ ${ }^{3}$ Uspensky Neuropsychiatric Boarding House \\ Porosskaya Street 1, 633146, Uspenka Village, Moshkovsky District, Novosibirsk Region, Russian Federation \\ ${ }^{4}$ Medical Center "Sineglazka" \\ Frunze Street 61, 630112, Novosibirsk, Russian Federation
}

\begin{abstract}
Background. Affective and addictive pathology among patients with schizophrenic spectrum disorders is not only widespread in the mentally healthy population, but also occupies a leading place in the structure of the schizophrenic process. Objective: to identify the features of addictive disorders and affective symptoms in schizophrenia. Materials and Methods. The study was carried out for two years (2019-2020). The main sample consisted of patients $(\mathrm{n}=34)$ with schizophrenia (F20) diagnosed according to ICD-10, with various clinical forms and duration of the disease, with the predominance of male patients $(n=32)$ compared to female patients $(n=2)$, mean age of the examined subjects was $28.5 \pm 9.8$ years. To diagnose affective symptoms, four standardized psychometric tools were used: The Hospital Anxiety and Depression Scale (HADS, Zigmond A.S., Snaith R.P., 1983), The Questionnaire for Determining the Level and Type of Loneliness (Korchagina S.G., 2008), Cook-Medley Hostility Scale (Ho Scale, Cook W.W., Medley D.M., 1954), The Beck Hopelessness Scale (BHS, Beck A.T. et al., 1974). The study of the propensity to addictions was carried out according to the Technique for the diagnosis of propensity to various addictions (Lozovaya G.V., 2007). Results. The high degree of propensity to three types of addiction (love, drug, healthy lifestyle), low and subclinical levels of anxiety and depression, as well as mild and moderate severity of hopelessness, deep experiencing the loneliness, moderate degree of propensity to aggression with the trend to low indices were revealed. Also, a reliable association was found between loneliness, religious dependence and sexual addiction.
\end{abstract}

Keywords: schizophrenia, psychiatric comorbidity, affective and addictive (addiction) disorder, anxiety, depression, hopelessness, loneliness, aggression and hostility.

\section{Received February 11, 2021}

Ovchinnikov Anatoly A. - MD, Prof, Head of the Department of Psychiatry, Narcology and Psychotherapy, Novosibirsk State Medical University, psychiatrist, addiction specialist, psychotherapist of the highest category, Novosibirsk, Russian Federation. SPIN-code 2228-5923. Author ID 705354. ORCID iD 0000-0003-1468-1620.

Sultanova Aklima N. - MD, assistant professor of the Department of Psychiatry, Narcology and Psychotherapy, Novosibirsk State Medical University, Novosibirsk, Russian Federation. ORCID iD 0000-0001-6420-6591.

Stankevich Anna S. - graduate student of the Department of Psychiatry, Narcology and Psychotherapy, Novosibirsk State Medical University, Novosibirsk, Russian Federation. ORCID iD 0000-0001-5627-8245. stankevichann97@ mail.ru

Narov Mikhail Yu. - MD, Prof. of the Department of Psychiatry, Narcology and Psychotherapy, Novosibirsk State Medical University, Novosibirsk, Russian Federation. ORCID iD 0000-0001-7551-6333.2996458@ mail.ru

Chut Ulyana Yu. - graduate student of the Department of Psychiatry, Narcology and Psychotherapy, Novosibirsk State Medical University, Novosibirsk, Russian Federation. ORCID iD 0000-0001-7991-374X. uu_chut@mail.ru

Ioanidi Diana K. - lecturer of the Department of Psychiatry, Narcology and Psychotherapy, Novosibirsk State Medical University, Novosibirsk, Russian Federation. ORCID iD 0000-0003-3103-2585. dion48@ mail.ru

Lugovenko Veronika A. - clinical psychologist, Medical Center "Sineglazka", Novosibirsk, Russian Federation. ORCID iD 0000-0003-3894-5029. veronika060596@mail.ru

Sultanova Aklima N., sultanova.aklima@yandex.ru 